\title{
Comprehensive Observation and its Role in Self-Awareness; An Emotion Recognition System Example
}

\author{
Nima TaheriNejad, Axel Jantsch, David Pollreisz \\ Institute of Computer Technology, TU Wien \\ Gusshausstrasse 27-29, 1040 Vienna, Austria \\ \{nima.taherinejad, axel.jantsch\}@tuwien.ac.at
}

\begin{abstract}
Observation plays a crucial role in self-awareness. In many scenarios, such as the Observe-Decide-Act (ODA) loops, self-awareness is founded upon observations of the system. In other words, observation generates the understanding of the system from the status and behavior of its self and its environment. Although recently more focus has been put on comprehensive and competent observations, we believe that further attention and work is due, especially in the field of cyberphysical systems. Hence, in this paper, we discuss our position on various aspects of observation methods. In a short list, the major aspects are Abstraction, Disambiguation, Desirability, Relevance, Data Reliability, Confidence, Attention, and History. We elaborate and anticipate the potential of these factors in improving the quality of the observation of the system, decreasing the processing load of higher layers, increasing the reliability of decisions, and consequently the overall performance of the system. To put these aspects into perspective, we elaborate them in the context of their potentials in our emotion recognition system under development.
\end{abstract}

\section{INTRODUCTION}

A COMMON requirement for many of the systems of today is an ability to perform correctly under a wide range of variation in their environment, as well as their internal states, parameters, applications and resources. Self-awareness is a feature that can enable these systems to show a robust and dependable behavior and meet their requirements.

Expecting 26 billion devices connected to the Internet of Things by $2020^{1}$ with an exponential growth trend means that manual maintenance, fault diagnosis and repair for all will soon be impossible [1]. This necessitates an embedded awareness of the system regarding its own state so that it detects and mitigates occurring faults. Self-awareness has been applied to both hardware [1] and software [2]. Some of the applications which have been explored for the implementation of self-aware concepts (under this term or other terms such as adaptivity, autonomy, goal-oriented and so on), are mobile applications [3], cloud computing [4], networks [5], operating systems [6], web [7], multi-core resource managers [8] and adaptive and dynamic compilation environment [9], (cyberphysical) system-on-chip [1], and health monitoring [10].

${ }^{1}$ www.gartner.com/newsroom/id/2636073
Several definitions for Self-awareness can be found in the literature [11], [1]. For instance, in [11], it is defined as "a system's ability to obtain and maintain knowledge about its state, behavior and progress." In [12], [1], it is defined as the ability of the system to "monitor its behavior to update one or more of its components to achieve its goals". These definitions highlight the fundamental importance of monitoring, or observation, in self-awareness of the system.

Observation is more than collecting data. Higher levels of hierarchy, or the core in centralized units, are often burdened with a large load of monitored data and the respective processing [13]. On the other hand, local monitoring also may be delayed due to the global communication traffic [13]. Therefore, to be a useful basis of self- and environmental awareness it has to be a process that filters, analyses, selects, abstracts, assesses, and actively ignores or requests sensory data. Moreover, observation is not limited to sensory data, but rather a more general concept which includes other observations of the system from its own and its parts, regarding performance [8], functionality or other factors. In some works, such as [14], [1], this duty is encapsulated in a unit called "Inspection Engine" or "Introspective Sentient Unit" in [12].

In brief, the observation process transforms raw data into a high-quality description of the system about itself and its environment. In the following sections we describe the various aspects and activities involved in the observation process, especially that of a Cyber-Physical System (CPS), discuss examples in an emotion recognition setting and finally summarize the observation process, its importance and its potential contributions to a self-aware system.

The remainder of this paper is organized as the following: In the next section, a brief review of a self-awareness architecture is presented, followed by a short review of usecases in Section III, where related works taking advantage of various aspects of a good observation are presented. Next, in Section IV, based on the aforementioned review of the existing literature, we present a summary of various aspects of a good observation scheme for self-aware CPSs. We clarify these concepts further in Section V, by producing an example in the context of an emotion recognition application which 


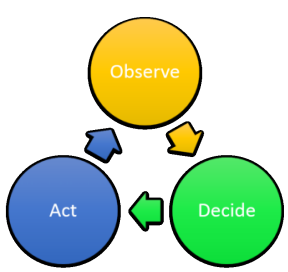

Fig. 1. Observe-Decide-Act (ODA) loop architecture for self-awareness

we have under development. Finally, Section VI concludes the paper.

\section{A Glance on the Architecture of SELF-AWARENESS}

Many architectures have been proposed in order to achieve self-awareness. In CPSs with limited resources available for self-awareness, such as SEEC ${ }^{2}$ [8] or Cyber-Physical Systemon-Chip (CPSoC) [1], an Observe-Decide-Act (ODA) architecture is frequently used. ODA is one of the prominent architectures for the CPSs with a central supervisor or control unit. For example, in the SEEC framework, an application specifies the goal and the system, based on its observation of the performance, chooses the best action from a list of possible actions, in order to meet the goals. CPSoC is distinguished from a Multi-Processor System-on-Chip (MPSoC) due to its observation capabilities [12]. In CPSoC observation is done across various hardware and software layers to monitor performance, decide on a proper action and steer actuators.

As seen in Fig. 1, the ODA loop starts with observation, showing its importance. However, in a considerable number of CPSs, sensory data are directly or with minimal processing fed to and used by the decision-making unit. The processing is also often a simple interpretation, abstraction, or transformation of the raw data and scarcely entails any contextual information about the data, how it is obtained, how reliable it is and how it should be interpreted. This implies an inherent trust in the hard-coded interpretation schemes, health, precision and accuracy of sensors, and the obtained sensory data. Therefore, a fault or failure in the sensory system or the collected raw data can easily propagate to higher levels of decision-making and consequently into actions. The same stands for environmental or contextual changes which do not comply with the hardcoded interpretation schemes. Therefore, as a countermeasure learning and prediction is used in some self-aware systems [11], [1].

Self-awareness, however, is not necessarily achieved only through a centralized control or supervisory unit. In some works such as [15], [16], [11], it is a cooperative or emerging behavior of the group of agents or subsystems. Nevertheless, attention and context-aware observation play a very important role in creating self-awareness. Preden et al. [15] describe the concept of context-awareness under situation-awareness which consists of values and interpretations of a set of situation

${ }^{2}$ SEEC is the name of the SElf-awarE Computing (SEEC) platform by Hoffman et al.[8] parameters. Situation parameters represent properties of a situation, processed and abstracted independently.

\section{OBSERVATION IN THE LITERATURE}

Traces of various aspects of a good observation can be tracked throughout the literature, although the terms, extent of usage, and thoroughness vary considerably. Thus, we base our position on a comprehensive observation of the literature, which provides us with good exemplars to follow in order to improve the performance of systems through improving their observation strategies. In this section, we briefly review some works in the literature which have emphasized or taken advantage of various aspects of a good observation scheme.

For example, in [8], the changes observed in the performance of the system are abstracted into heartbeats (an abstracted notion of time) which provide information about the execution of the application. This abstraction enables the framework to be adopted and used by various programs where the nature of goals are significantly different. All that is needed for this adoption is presenting the performance goal in the heartbeat abstraction notion. Moreover, available actions and reasoning in the decision-making process are abstracted into their effect on the speed of the system. Hence, SEEC serves as an example to show that abstraction does not necessarily need to be bottom up, and it can be used in top-down observations as well. We note that bottom-up abstraction often reflects the perception of the system from its environment, whereas top-down observations normally reflect the perception of the system about the performance of some parts of the system, or the system as a whole.

In [11], abstraction is done through online learning instead of predefined knowledge and rules. This increases flexibility and resilience of the overall system. In CPSoC [1], a virtual sensing platform is used both for the purpose of abstraction and disambiguation in the case of sensor fusions or faults and errors.

In [17] the authors have tried to create a unified desirability scale to compare and prioritize parameters of different nature which are directly related or comparable. A form of desirability scale is also found in [18].

Rinner et al. [11] briefly point to the selection of an object tracking algorithm which has "an acceptable level of robustness", which approves of the importance of confidence, which is dependent on the reliability of other observations. Hoffman et al. [8], on the other hand, use the correlation of the recent past and near future, to assess the accuracy of the control scenario. Moreover, changes in this factor are considered in -and propagate to- the decision-making process. In CPSoC [1], a lifetime reliability characterization matrix is kept in the OS layer, which uses the data from reliability sensors in order to balance the workload of different units and thus increase the overall reliability during the lifetime.

The quality of sensors and measurements are in many systems unknown and cannot be guaranteed for the whole lifetime of the system. Therefore, data reliability analysis should always be an integral part of comprehensive observations. 
In [11], for assessing the degree of the confidence of their observation (finding and tracking objects of interest), they use a measure of similarity. In this study, an existing database enables application of this procedure to obtain a figure of confidence. Alternatively, in some other systems history could replace this database or provide measures of confidence with other methods. However, self-assessment of the confidence by the system itself remains a major challenge in many cases. Specifically considering that a supervised assessment or validation of the performance of the system is not always possible, and often it is not desirable. Lack of history can increase the difficulty of autonomic acquisition of this aspect (confidence).

In [8], the history of recent observations are used to model the behavior of the system and predict whether a change in behavior is necessary, and if so, in what form. Typically, larger tracks of history can help in obtaining a better and more confident prediction of the future. However, maintaining a large memory is often challenging, in terms of storage and its respective costs, as well as the hardship and computation costs of processing a large amount of data. Therefore, it is important to use creative methods of storing data; that are compacting the data in such a way that necessary space decreases while valuable information is kept. Abstracting the data into the aspects mentioned above can help in achieving this goal.

\section{Aspects of A COMPREHENSIVE OBSERVATION}

Building on the observation strategies in various related works (some of which were reviewed in Section III), in this section we present our position on various aspects of data interpretation which can lead to a good and comprehensive observation. Specific systems may not need or be able to afford all the required resources for such implementation as it will follow. Therefore, based on the details of the case, these aspects should be prioritized and used according to the case at hand.

\section{A. Abstraction}

1) Definition: Appropriate selection of the representation of the information in order to obtain compact knowledge, relevant to a particular purpose.

2) Description: Although collecting more data can help in improving the awareness and thus the performance of the system -since this relationship is not linear [14]- it does not guarantee that. To balance the processing load at various levels of the hierarchy, data need to be properly abstracted, which implies a meaningful mapping of the measured values to properties. A well-defined format is also crucial for a good abstraction, especially in distributed and hierarchical observation schemes [13].

3) Example: One of the parameters that is often measured in many e-health systems -and in our emotion recognition system as well- is the heartbeat rate. This value with all the potential noise and variations could be passed on to the central system for processing and decision making. Alternatively, these values can be mapped to some abstracted values (e.g., only five values of extremely low, low, normal, high, and extremely high) and thus transferred with smaller communication burden while decreasing the processing load of the decisionmaking unit.

4) Challenges: Predefined abstraction scenarios show little flexibility. While meaningful for a CPS in a well-defined environment with clear and stable objectives, it can cause an impediment for an autonomous system in following its evolving needs to adapt to new environment and situations.

5) Potential Solutions: As a solution, the system itself can create the most appropriate abstract properties based on unsupervised learning processes (for an example see [11]). This solution is significantly more challenging to implement and requires considerable resources. Nevertheless, it appears to be a prerequisite for truly autonomous and evolving systems.

\section{B. Disambiguation}

1) Definition: Remove uncertainty of meaning from measured data, or resolving conflicts arisen by different data.

2) Description: Despite a good abstraction scenario, the data can often be interpreted in more than one way or different parts of the system may have a different (conflicting) experience of the environment which could be natural or due to faults and failures. In either case, a functional, well-defined disambiguation strategy is a necessity. This prevents propagation of ambiguities or misinterpretations and thus facilitates decision making in the system.

3) Example: Heartbeat or temperature sensor may come from a chest belt, a smart watch or other sensors with various sensitivity and accuracy. Given information on data reliability (including accuracy or precision) may help in arbitrating nonmatching values, e.g., heartbeat information from chest belt is more accurate than the one from a smart watch, hence the value of chest belt should be typically considered as the reference value, if the numbers are not matching.

4) Challenges: One of the challenges for disambiguation is again flexibility. For example, it is possible that the user is not wearing the chest belt and only noisy random values are observed, or for reasons of aging of the device or faults the accuracy of the chest belt has degraded. In such cases, the system should be able to change its arbitration and use alternative and more reliable values.

5) Potential Solutions: Parameters such as History, Confidence and Data Reliability of the measurements can help in identifying a need for a change in the disambiguation strategies and adapt the right alternative. A sudden drop in values can show taking off the chest belt (History), variations larger than usual, could imply some degradation (leading to decreased confidence) and loss or malfunction of some sensors in the chest belt constructing the final measurement could indicate less reliable measurements (data reliability).

On a higher level, and in a top-down flow of information, contextual awareness and predictions can provide meaningful inputs to the disambiguation unit as well. For example, is a low or high heartbeat accompanied by a low or high body temperature? Or, if the person has started or stopped a sport 
activity, considerable and synchronized increase or decrease in heartbeat and temperature are expected (which do not reflect the feelings of the user).

\section{Desirability}

1) Definition: The quality of being inline with achieving one (or more) of the goals or expected outcomes of the system.

2) Description: Desirability is an evaluation of the system regarding the state of its own components and their alignment to its goals and expectations. When inferred states are marked as desirable or undesirable (possibly on a scale to distinguish between more and less desirable situations), a value system is implied that is rooted in the objectives and purpose of the system. If the system has only one sole purpose, the mapping of properties and states onto a desirability scale is less necessary. However, if the system pursues potentially contradicting goals a mapping onto a desirability scale is a useful intermediate step for resolving conflicts as well. A desirability scale serves as a unifying currency that allows for the comparison of otherwise unrelated properties and states.

3) Example: Let us assume the system is confronted with a discrepancy in the incoming data stream while on a low battery. The system has to decide if it is more important to preserve energy or to forward the detected anomaly for further processing. In such cases, a " 1 " or " 0 " bit on whether a discrepancy has happened or not, or the battery is full or low provides insufficient information for making a suitable and well-informed decision. If these events (observations) are mapped onto a desirability scale, this additional aspect of observation can show how undesirable a discrepancy has hap pened and how desirable would forwarding this problem be Given the continuous level of charge in the battery (desirability of the available power), the system has further knowledge and flexibility in making an informed decision inline with its goals, for such situations where observations are not directly comparable or related.

4) Challenges: Creating an appropriate mapping of desirability for possible states of the components is sometimes very challenging. Particularly, when there is no direct link between the state of the component to be mapped to the desirability scale and the goals and expectations of the system.

5) Potential Solutions: One solution could be evaluating the effect of possible states of that component on achieving the goals of the system, via proxies and/or in isolation. However, this task itself (isolating the effect of the states of a nondirectly linked component in achieving the goals), can be considerably challenging.

\section{Relevance}

1) Definition: The quality of being closely connected to/important for the matter at hand.

2) Description: Relevance has similarities with desirability, however, rather than states, it regards measurements and values or parameters and variables. Moreover, relevance regards smaller details in the system; that is, instead of considering alignment with the overall goals and expectations of the system, it considers the importance and connection of a measured value or parameter to a certain specific analysis in the system. This aspect can help in finding the right balance in the weight of a parameter in disambiguation, conflict resolution or decision-making as well as resources dedicated to that observation. This not only offloads the decision-making unit but also it enriches the observation unit by providing it with information on where more attention is necessary. Attention can affect how the raw data are obtained (such as the degree of redundancy, or frequency of data collection), or how it is processed, e.g., using precise methods requiring larger resources or less precise but lighter methods (such as approximate computing).

3) Example: Our preliminary measurements show that Anger and Sadness, for example, have a strong correlation with slow decrements of skin temperature, very weak correlation to the heartbeat and moderate to weak correlation to skin conductance, also known as Electro-Dermal Activity (EDA). This means that relevance of skin temperature to the two feelings is significantly higher than the other two. Hence, if the skin conductance and temperature are giving conflicting signals, skin temperature will be given greater weight in determining whether the feeling is one of the two or not.

4) Challenges: Traditionally, relevance is set by the designer, at design time. However, the relevance could change by the way of extracting it from a larger set of data. Once the designer does that, program upgrades are released. Nonetheless, this does not take into account personalization of this factor regarding the biometrics of the particular user.

5) Potential Solutions: The relevance of a parameter could be initialized, or predefined. Ideally, however, the system can learn the trends and change this value based on its experience and adapt it to its current situation and the biometrics of the specific user.

\section{E. Data Reliability}

1) Definition: the extent to which a measuring procedure yields the same results on repeated trials.

2) Description: As important as the data themselves, is the knowledge about its thoroughness, accuracy and precision, which allows the system to perform (here to observe/monitor) within the expected limits and stated conditions. Here accuracy describes the systematic bias of the data values compared to the real values of the measured quantity ${ }^{3}$, and precision denotes the random errors in repeated measurements under the same conditions ${ }^{4}$. Although the concept of measurement device precision and propagation of inaccuracies into the system through calculations is well established, more attention by CPS engineers is due in system design ${ }^{5}$.

3) Example: Awareness of the system about the robustness of the given values and their data reliability in certain situations can help the system to choose the best method for

\footnotetext{
${ }^{3}$ In other words, accuracy is a measure of statistical bias.

${ }^{4}$ In other words, precision is a measure of statistical variability.

${ }^{5}$ Although in approximate computing this matter is well considered, we believe that it should receive more attention in exact computing as well.
} 
each situation (in the case of multi-mode technology) without needing to have an in-depth analysis of the situation and speculations on the accuracy of the given systems. That is, e.g., relying primarily on the heartbeat values coming from the chest-belt for precise information, before the smart watch and other means. Of course, if the data reliability of the chest-belt has been set to lower values (compared to the smart watch or other means), or if a problem, conflict or discrepancy has been already observed, the data with the highest reliability should be considered as the main/primary reference for the values.

4) Challenges: Propagation of measurements errors through the system, specifically, when several post-processing steps are taken, can impact the data reliability of the final assessment and hence the decision of the system concerning its situation. Whether the information is current or updated when it reaches the decision unit, or when a decision is reached, is another concern regarding the data reliability.

5) Potential Solutions: One solution is taking parameters partaking in the data reliability and robustness of measurements into account during the system design. These parameters could be the type of sensors and measurements (and its inherent accuracy and precision limits), bias set-up and peripherals of the sensory system, type and size of postprocessing, validity period of the measured data, and the health (functionality) of the sensors themselves which could be affected by aging, or the environment.

We also note that one method to increase the data reliability is redundancy. Redundancy allows to collect the same data in different ways, for instance with various sensors, and to compare the consistency of the different, independent sources. One method of checking consistency is following the trend of changes in a single set of data and comparing the values with possible or impossible variations in the trend and data values. The other method is comparing different data sources and analyzing them for their mutual consistency.

\section{F. Confidence}

1) Definition: the extent to which a procedure may yield the same results on repeated trials.

2) Description: Confidence has significant similarities to data reliability, however, rather than measured data (or propagated results based on closed-form analysis), it regards experiments and procedures where a comprehensive external verification of accuracy and precision is not performed. This determines the firmness or flexibility of the system concerning its learning, analysis and decisions. In other words, to what extent the learned trends, analysis and decisions should be relied upon, or alternative analysis should be prepared or further data processed.

3) Example: Whether the temperature sensor is attached to the body or not, is not a measure of reliability. The confidence of the system in its attachment to the body, however, can affect how the system reacts to values out of the norm. To this end, a top-down variant of consistency analysis can be used. That is, a comparison of the measured values with expectations. Body temperature hardly undergoes a large change within seconds (or minutes). Therefore, a sudden drastic change could show a consistency problem and decrease the confidence of the system with regard to the measured value ${ }^{6}$. However, how reliable is consistency checking for assessing the attachment of the sensor is itself subject to the question of reliability (which constitutes the confidence of the system in this procedure). Should the system rely on this analysis or use other methods, such as asking the user?

4) Challenges: Creating a confidence measure, assessing or improving it can be a very challenging task, especially when it is to be unsupervised or with minimum external inputs. To be more specific, creating a fairly reliable (unsupervised) selfassessment procedure can be extremely hard.

5) Potential Solutions: Confidence can be built up based on the reliability of the inputs and the history of analysis. That is, analyzing the consistency of decisions, previous analysis, and the outputs given the data reliability of the inputs. One method of checking confidence is following the trend of outputs obtained by different procedures given a single set of data and comparing the values with each other and if possible, with the correct or desired values. The confidence values, in turn, can drive, how heavy a given procedure weighs in the interpretation of the current situation.

\section{G. Attention}

1) Definition: Selective allocation of limited resources to specific tasks.

2) Description: It is neither meaningful nor efficient to always collect and process all possible data. Attention decides on which observations to allocate the available communication, computation, memory and energy resources. The observation process should be guided by an understanding of what data are required for a given purpose in a given situation. At the same time, attention allows a low effort, continuous scanning of all sensory input to detect unexpected events and anomalies. If the attention process suspects the arrival of important new data, it focuses the attention, i.e. allocates the resources, on their collection and analysis. From these considerations, we infer that attention is driven both bottom-up, by the incoming data, and top-down, by prevailing expectations and goals.

3) Example: Using attention the system can, based on aspects such as relevance for example, optimize the resource allocation for data collection. As we present the details of our measurements in Section V, EDA has some correlation (weaker or stronger) with all targeted emotions. Therefore, instead of processing all measured data, EDA can be monitored and once an activity is observed there -based on the relevance to the suspected feeling- other processes can be activated selectively. Thus reducing the processing load of the system and optimizing resource utilization.

4) Challenges: In order to trigger attention in the observation process, the system should be able to detect certain trends and anomalies. A task which can be challenging given

\footnotetext{
${ }^{6}$ This, in turn, can trigger further processes which can determine whether this consistency problem is due to a normal environmental change (i.e., the sensor is detached) or a fault or failure.
} 
the wide range of data that can be observed as well as trends or anomalies which can occur and need to be detected. However, using such methods for observatory data streams implies availability of very strict and limited resources which can render many prominent methods impractical. In addition, once an event is observed in a data stream which triggers further processing, other data which were not part of the focus of the system may be necessary and potentially missed.

5) Potential Solutions: A potential countermeasure for missing unfocused data when an event is detected and change of attention is triggered, could be using local buffers. Deciding the length of this buffer and its required resources can impose certain restrictions on the designer. Various learning methods and smart anomaly detection systems are potential solutions for detecting events in the data stream. However, we note that creating efficient methods with extremely limited resources is still a challenge.

\section{H. History}

1) Definition: Recording and studying a series of past events connected to an entity.

2) Description: History allows extraction of statistics from a series of observations; assessing the performance of the system over time, its improvements or deterioration, assessment of the quality of sensory data over time, and changes of the environment over time. Based on such historical data and analysis, the system is given the capability to predict its own performance in the near future, the expected failure of sensors and actuators, and future trends in the environment. In turn, this understanding can motivate the system to evolve its own goals, strategies, and tactics in order to adapt to these observations and predictions.

3) Example: History can play a crucial role in many different aspects, such as confidence (through consistency analysis, as mentioned above). It can increase relevance by establishing new correlations between factors that were not predetermined, however, their correlation is discovered during the process. In other words, what feelings correspond better to which measurements. For example, is happiness better associated with skin temperature or skin conductance? More importantly, history can help in determining the standard state of the system and identify potential abnormalities, which is a parameter tightly coupled with predictions. For example, history can establish what is the normal heartbeat rate of a person. This sets the expectation of the system on the heartbeat rate and its changes for each feeling.

4) Challenges: The greatest challenge of this aspect is the limited available memory. How to abstract, compact, and archive the data are some of other problems which often are affected by the limited memory as well.

5) Potential Solutions: By history, we do not necessarily imply an explicit bank of historical data. History can be inherent to some other parameters and implied rather than explicit with formal appearances. An instance is the average value or the trends of change and evolution of a parameter which may not include a bank of historical data to back the trend but does represent history. Another instance could be events and trends of their occurrence rather than the actual data. Periodically compacting and forgetting (eliminating old or less relevant data) are some other potential solutions.

\section{COMPREHENSIVE OBSERVATION FOR OUR EMOTION RECOGNITION SYSTEM}

To validate helpfulness of a comprehensive observation as discussed in Section IV, we plan to implement those aspects in an emotion recognition system. In this section, we try to clarify these concepts further by the way of examples in our design for our emotion recognition system under the development. Here, we suggest how the aforementioned aspects can be tailored, taken advantage, implemented and improve the "observe" unit of the system and consequently overall performance of the system.

\section{A. Set-up}

For our emotion recognition system, we use Empatica, "E4 Wristband" smart watch which has the following sensors embedded: A photoplethysmogram (PPG) to measure Blood Volume Pulse (BVP), Heartbeat and Heart Rate Variability (HRV), Electr-Dermal Activity (EDA) sensor for skin conductance, infrared thermopile for skin temperature, and 3-axis Accelerometer for capturing motions.

For measurements, subjects were asked to wear the smart watch and remain seated for one minute, so that we could obtain their baseline. To solicit Sadness, Happiness, Anger and Fear, short videos were played -in a random order- for the subjects. Afterwards, the subjects were asked to remain seated for one more minute (to get back to their baseline), while filling a self-assessment form, reporting the emotion they felt and how strongly they felt it.

Measured data was collected and plotted in Matlab. In the future, this data needs to be post-processed and the emotions extracted on some hardware with very limited resources (such as a smartphone). For this reason, limited available resources is one of the primary constraints in this system, and we will describe how we plan to exploit comprehensive observation to increase the efficiency of the system.

\section{B. Preliminary Measurements}

Four participants, male, between the age of 20 and 25, took part in our preliminary measurements. Fig. 2 shows a sample, namely the measured EDA signal, during two different experiments: happiness and sadness. We observe that when the subject experiences the feeling during the video (between the star marks), his EDA changes with two different trends. For happiness, this trend appears as (relatively) large repetitive peaks, whereas as for sadness this seems to be a slow increment. Studying other measurements obtained from other subjects, the absolute value/level of EDA seems to carry no considerable significance in identifying these emotions. Therefore, an algorithm needs to be developed which can identify such trends in the EDA signal and notify the 'Decide' unit (see Fig. 1) of the system. 


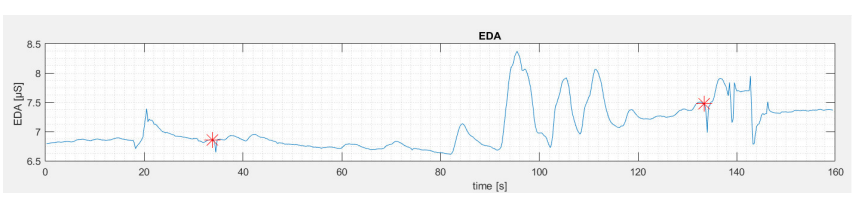

(a) Happiness

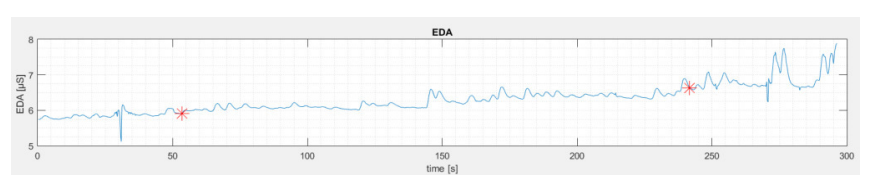

(b) Sadness

Fig. 2. Skin conductance (EDA) Signal for (a) Happiness, and (b) Sadness.

One of the simplest solutions to this problem is to define templates based on our measurements and compare the incoming data with the template. In this case, on top of identifying a matching/non-matching trend, the degree of similarity of the data with the template (how matching are the data compared to the template), can be used as the confidence of that unit in identifying the trends it is looking for. Hence, if a trend in the data is identified (or rejected), and thus causes a conflict in higher levels of the system, by looking into the confidence aspect, the potential source of conflict can be traced and the conflict can be resolved.

Table I summarizes the trends we have observed in our measurements. In this table, the background color of cells shows the relevance of that trend to the respective emotion. The lightness of the cell background shows how likely it is that with such emotion, such a trend in the measured parameter may be observed. To associate a level of relevance to each factor we have used the likelihood or unanimity of occurrence in our experiments. For example, in our measurements unanimously- shortly after a person sees a scary video, their heartbeat increases. Therefore, we can confidently associate this observation with this feeling, and consider it highly relevant. On the other hand, a peak in EDA was not observed in every case, due to which we are less confident in our conclusion regarding the relevance of a single short peak in skin conductance, to the fear. Therefore, we consider it slightly less determinant for this feeling, and hence less relevant.

Our primary measurements on heartbeat values also show that only its changes from the baseline are relevant (rather than its absolute value/level). So the heartbeat can be abstracted to four values of increasing, decreasing, rise and fall, and constant (as shown in Table I). The regular heartbeat rate however, varies with the person under study. This variation

TABLE I

SUMMARY OF OBSERVED TRENDS IN OUR MEASUREMENTS.

\begin{tabular}{|c|c|c|c|}
\hline & Skin Conductance & Skin Temperature & Heartbeat Rate \\
\hline \hline Happiness & Repetitive Peaks & Slow Increment & Constant \\
\hline Sadness & Slow Increment & Slow Decrease & Rise and Fall \\
\hline Fear & A short Peak & Constant & Small Increment \\
\hline Anger & No Correlation & Slow Decrease & Constant \\
\hline
\end{tabular}

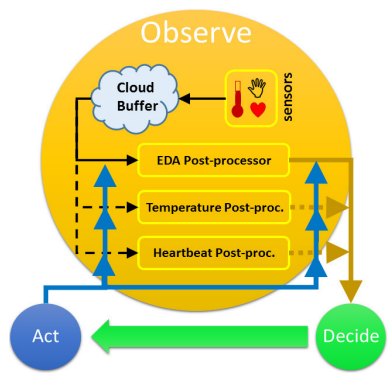

Fig. 3. Details of the "Observe" unit for our emotion recognition system.

should be considered when the person's emotion is being analyzed. To this end also, a small footprint learning method -based on the history- can be employed in the system. For the learning process, often feedback from higher levels (after categorization of emotions) are required and beneficial. Therefore, exchanging abstracted data needs to happen not only in a bottom-up manner but also in a top-down approach.

Based on such arguments and similar design analysis acquired during our preliminary measurements (a summary of which we have inserted in Table I), we propose the following system architecture for the "Observe" unit of our emotion recognition system. In this system we try to -whenever possible and beneficial- take advantage of as many observation aspects as possible.

\section{System Architecture}

For our system, we consider an ODA loop architecture. In this section, we further explain the details of the "Observe" unit in the architecture. As seen in Fig. 3, observation starts with collecting sensory data which are buffered on the cloud. This data need to be post-processed, in the following units.

To be efficient in communication and processing power, we take advantage of attention. That is, instead of continuously processing all data, they are periodically processed. To decrease the odds of missing an event, one data stream is considered as the default and is continuously processed. As mentioned in Section IV and seen in Table I, skin conductance (EDA) shows the most sensitivity to emotional experiences. Therefore, this stream is the default stream which is continuously processed in order to find potential events. Once an event is observed by the EDA post-processing unit, it triggers the "Decide" unit, which, based on the event and suspected/possible related emotion(s), can decide what other information is necessary. Accordingly, the action unit activates the respective post-processing unit(s) to acquire the relevant data from the cloud and process them. We note that the postprocessing units in Fig. 3 are logical units and thanks to the attention based operation, the hardware itself could be shared.

Further details of the generic post-processor design are shown in Fig. 4. The most challenging unit in the postprocessor is the matching unit where certain trends (according to Table I) are looked for and if found, abstracted data (abstracted following the trends categories listed in Table I) informs higher levels (decision unit) about them. Alongside 


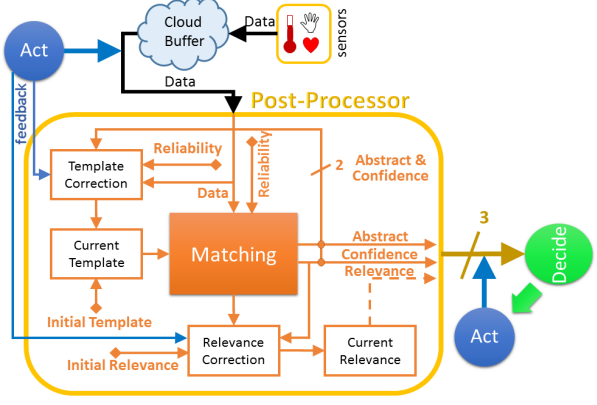

Fig. 4. Details of the post-processor unit.

the abstracted data, the confidence level of the findings can be sent to decision unit to facilitate the process of decisionmaking. Confidence information can be sent by the request of other units (such as when they need it for disambiguation or conflict resolution) or constantly with the abstract.

Relevance information on the other hand, undergoes very little and infrequent changes and therefore is sent only when a change in its value is observed in the post-processor unit (thus shown by a dashed line). Observing such a change necessitates keeping a history which is kept in the "relevance correction unit". This unit is initiated by the designer (represented as the background color of each cell in Table I) and updated by the feedback from higher levels of hierarchy (which includes information on the confidence of the given feedback) for each matched trend and taking into account the respective confidences. A similar procedure transpires for updating the template which is used for finding the patterns. We note that for template correction -similar to matching unit- the reliability of sensory data is also used. A parameter which is provided by the designer at design time or provided automatically by the sensors, once attached.

\section{CONCLUSION}

The importance of the observational process can hardly be overrated. In addition to the bare values, measured data have many contextual aspects that determine what it means and how it should be used. This host of information around the collected data is the basis of assessing the state of a system, its performance, its environment, and the influences of its actions.

Observation can be both a bottom-up and top-down process. The measured data are usually collected, processed, analyzed and abstracted bottom-up, while top-down expectations, hypotheses and needs greatly influence, steer, activate or block these bottom-up processes. Information redundancy and consistency analysis have to be used to assess confidence and reliability of data and lead to an appropriate assessment of the situation. Event-driven resource allocation (attention), triggered bottom-up or top-down, is another aspect which can considerably off-load the processing and communication.

After in-depth discussions on various aspects of a good and comprehensive observation, we described how we plan to use these concepts in our emotion recognition system under the development. Although these concepts can be employed, in "Decide" and "Act" units as well, since the role of a comprehensive observation is bolder in the "Observe" unit, we described the details of our comprehensive observation plan only for this unit of our system.

\section{REFERENCES}

11] N. Dutt, A. Jantsch, and S. Sarma, "Toward smart embedded systems: A self-aware system-on-chip (SoC) perspective," ACM Transactions on Embedded Computing Systems (TECS), vol. 15, no. 2, p. 22, 2016.

[2] J. O. Kephart and D. M. Chess, "The vision of autonomic computing," Computer, vol. 36, no. 1, pp. 41-50, 2003.

[3] P. Mercati, A. Bartolini, F. Paterna, T. S. Rosing, and L. Benini, "A linux-governor based dynamic reliability manager for android mobile devices," in Design, Automation and Test in Europe Conference and Exhibition (DATE), 2014. IEEE, 2014, pp. 1-4.

4] B. Jennings and R. Stadler, "Resource management in clouds: Survey and research challenges," Journal of Network and Systems Management, pp. 1-53, 2014. [Online]. Available: http://dx.doi.org/10 1007/s10922-014-9307-7

[5] P. eth ee Spathis and M. Bicudo, "ANA: Autonomic network architecture," Autonomic Network Management Principles, p. 49, 2011.

[6] L. Wanner, S. Elmalaki, L. Lai, P. Gupta, and M. Srivastava, "VarEMU: An emulation testbed for variability-aware software," in Hardware/Software Codesign and System Synthesis (CODES+ISSS), 2013 International Conference on, 2013, pp. 1-10.

[7] J. Strassner, S.-S. Kim, and J. W.-K. Hong, "The design of an autonomic communication element to manage future internet services," in Management Enabling the Future Internet for Changing Business and New Computing Services. Springer, 2009, pp. 122-132.

[8] H. Hoffmann, M. Maggio, M. D. Santambrogio, A. Leva, and A. Agarwal, "SEEC: A framework for self-aware computing," MIT, Cambrige, Massachusetts, Tech. Rep. MIT-CSAIL-TR-2010-049, October 2010.

[9] W. Baek and T. M. Chilimbi, "Green: a framework for supporting energy-conscious programming using controlled approximation," in ACM Sigplan Notices, vol. 45, no. 6. ACM, 2010, pp. 198-209.

[10] J.-S. Preden, K. Tammemäe, A. Jantsch, M. Leier, A. Riid, and E. Calis "The benefits of self-awareness and attention in fog and mist computing," IEEE Computer, Special Issue on Self-Aware/Expressive Computing Systems, pp. 37-45, July 2015.

[11] B. Rinner, L. Esterle, J. Simonjan, G. Nebehay, R. Pflugfelder, G. Fernandez Dominguez, and P. R. Lewis, "Self-aware and self-expressive camera networks," Computer, vol. 48, no. 7, pp. 21-28, 2015.

[12] S. Sarma, N. Dutt, P. Gupta, A. Nicolau, and N. Venkatasubramanian, "Cyberphysical-system-on-chip (CPSoC): A self-aware MPSoC paradigm with cross-layer virtual sensing and actuation," in Proceedngs of the Design, Automation and Test in Europe Conference and Exhibition (DATE), Grenoble, France, March 2015.

[13] L. Guang, J. Plosila, J. Isoaho, and H. Tenhunen, "Hierarchical agen monitored parallel on-chip system: A novel design paradigm and its formal specification," International Journal of Embedded and Real-Time Communication Systems (IJERTCS), vol. 1, no. 2, 2010.

[14] A. Jantsch and K. Tammemäe, "A framework of awareness for artificial subjects," in Proceedings of the 2014 International Conference on Hardware/Software Codesign and System Synthesis, ser. CODES '14. New York, NY, USA: ACM, 2014, pp. 20:1-20:3. [Online]. Available: http://jantsch.se/AxelJantsch/papers/2014/AxelJantsch-CODES.pdf

[15] J.-S. Preden, J. Llinas, G. Rogava, R. Pathma, and L. Motus, "On-line data validation in distributed data fusion," in Ground/Air Multisensor Interoperability, Integration, and Networking for Persistent ISR IV: SPIE Defense, Security and Sensing, T. Pham, M. A. Kolodny, and K. L. Priddy, Eds. SPIE - International Society for Optics and Photonics, 2013

[16] J.-S. Preden, "Generating situation awareness in cyber-physical systems: Creation and excahnge of situational information," in Proceedings of the 2014 International Conference on Hardware/Software Codesign and System Synthesis. New York, NY, USA: ACM, October 2014.

[17] M. Sánchez-Escribano and R. Sanz, "Emotions and the engineering of adaptiveness," in Procedia Computer Science: Conference on Systems Engineering Research, vol. 28. Madrid, Spain: Elsevier, 2014, pp. $473-480$.

[18] H. Hoffmann, "CoAdapt: Predictable behavior for accuracy-aware applications running on power-aware systems," in Real-Time Systems (ECRTS), 2014 26th Euromicro Conference on, July 2014, pp. 223-232. 